\title{
Incidence and Prognostic Factors for Legionnaires' Disease in Denmark 1993-2006
}

\author{
G. St-Martin, ${ }^{1}$ S. Uldum, ${ }^{2}$ and K. Mølbak ${ }^{1}$ \\ ${ }^{1}$ Department of Epidemiology, Statens Serum Institut, Ørestads Boulevard 5, 2300 Copenhagen S, Denmark \\ ${ }^{2}$ Department of Microbiological Surveillance and Research, Statens Serum Institut, Ørestads Boulevard 5 , \\ 2300 Copenhagen S, Denmark
}

Correspondence should be addressed to G. St-Martin; gry.stm@gmail.com

Received 4 May 2012; Accepted 10 June 2012

Academic Editors: A. Jin-xia Zhang and L. Lillienberg

Copyright (c) 2013 G. St-Martin et al. This is an open access article distributed under the Creative Commons Attribution License, which permits unrestricted use, distribution, and reproduction in any medium, provided the original work is properly cited.

\begin{abstract}
Objectives. To describe the incidence of Legionnaires' disease and factors affecting survival in Denmark from 1993 to 2006 . Methods. Retrospective register study using data on episodes of Legionnaires' disease from notification forms and the National Reference Laboratory, and information on comorbidity from national registers on discharge diagnoses and mortality. Results. The average annual incidence of laboratory confirmed cases was 1.5 per 100000 people. The 30 -day case fatality was $19 \%$, ranging from $7 \%$ in travellers to $37 \%$ in hospital-acquired cases. Patients older than 60 years and patients with underlying disease were more likely-to die, as were patients infected with L. pneumophila serogroup 6. The lowest mortality was seen for L. pneumophila serogroup 1. Conclusion. Increased awareness of the disease, better diagnostics, and appropriate testing and treatment strategies are needed to improve survival. In particular, we focus on the relevance of diagnostic methods, such as polymerase chain reaction (PCR), that detect other serogroups than the urinary antigen tests used that will only reliably detect antigen from L. pneumophila serogroup 1 . PCR is faster and more sensitive than culture.
\end{abstract}

\section{Introduction}

The term Legionnaires' disease refers to pneumonia caused by legionella bacteria. When the disease was first described about 30 years ago, it was perceived as a very severe but rare form of pneumonia with a high case fatality rate (CFR) of at least $15 \%$. Since then, several studies have shown that Legionnaires' disease has a much broader spectrum, ranging from mild pneumonia in out-patients to the originally described severe disease, and that legionella is one of the most common causes of community acquired pneumonia requiring hospitalization [1-4]. More than 50 legionella species have been described, of which approximately 20 are known to have caused human disease, as they have been isolated in samples from pneumonia patients. However, the far most common cause of Legionnaires' disease is L. pneumophila and especially serogroup 1 . This is also the one most easily diagnosed because of the existence of the urinary antigen test (UAT) for legionella, which has the highest sensitivity for $L$. pneumophila serogroup $1[1,2,5]$.
In Denmark, Legionnaires' disease has been notifiable by treating clinicians to the Department of Epidemiology at Statens Serum Institut since 1980. In parallel, the National Reference Laboratory at Statens Serum Institut performs diagnostics and collects isolates of legionellae from the local clinical microbiology laboratories for species identification and serogrouping.

The aims of the present study were to describe the incidence of and survival following Legionnaires' disease in Denmark, to analyse the outcome of infection with different species and serogroups and according to various patient factors.

\section{Materials and Methods}

The study was a retrospective, register-based analysis of confirmed, notified cases of Legionnaires' disease, defined as pneumonia caused by legionella, in Denmark between 1993 and 2006, the period for which morbidity and mortality 
TABLE 1: National laboratory criteria for confirmed cases.

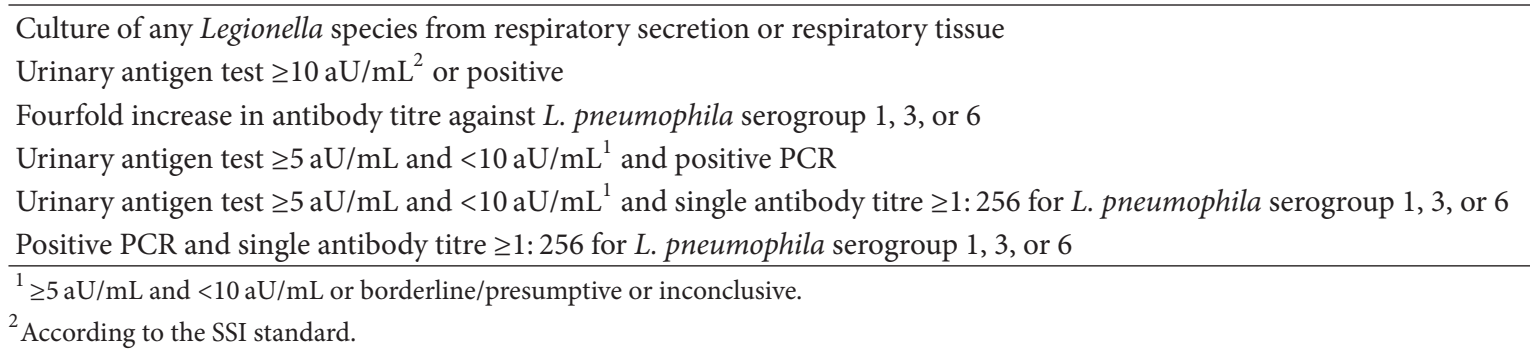

TABLE 2: Number and proportion of patients in each comorbidity group for different exposure categories; notified, confirmed cases of LD, Denmark 1993-2006, $N=1116$.

\begin{tabular}{|c|c|c|c|c|}
\hline Comorbidity group & All patients number $(\%)$ & $\begin{array}{c}\text { Travel associated } \\
\text { number }(\%)\end{array}$ & $\begin{array}{c}\text { Hospital acquired } \\
\text { number }(\%)\end{array}$ & $\begin{array}{l}\text { Community acquired, } \\
\text { nontravel number (\%) }\end{array}$ \\
\hline Alcohol related diagnoses & $45(4.0 \%)$ & $8(2.7 \%)$ & $7(4.2 \%)$ & $30(4.6 \%)$ \\
\hline Cardiovascular diseases & $332(30 \%)$ & $65(22 \%)$ & $84(51 \%)$ & $183(28 \%)$ \\
\hline Pulmonary diseases & $376(34 \%)$ & $74(25 \%)$ & $67(40 \%)$ & $235(36 \%)$ \\
\hline Immune deficiency diseases & $50(4.5 \%)$ & $9(3.0 \%)$ & $21(13 \%)$ & $20(3.1 \%)$ \\
\hline Infectious diseases $^{1}$ & $153(14 \%)$ & $29(10 \%)$ & $38(23 \%)$ & $96(13 \%)$ \\
\hline Neurological diseases & $42(3.8 \%)$ & $7(2.3 \%)$ & $10(6.0 \%)$ & $25(3.9 \%)$ \\
\hline Renal diseases & $205(18 \%)$ & $42(14 \%)$ & $46(28 \%)$ & $117(18 \%)$ \\
\hline Gastroenterological diseases & $193(17 \%)$ & $39(13 \%)$ & $53(32 \%)$ & $101(16 \%)$ \\
\hline Diabetes & $104(9.3 \%)$ & $32(11 \%)$ & $13(7.8 \%)$ & $59(9.1 \%)$ \\
\hline Malignancy & $150(13 \%)$ & $18(6.0 \%)$ & $57(34 \%)$ & $75(12 \%)$ \\
\hline Others $^{2}$ & $230(21 \%)$ & $31(10 \%)$ & $67(40 \%)$ & $132(20 \%)$ \\
\hline
\end{tabular}

${ }^{1}$ Excluding Legionnaires' disease.

${ }^{2}$ Rheumatologic diseases, endocrine diseases (except diabetes), nonmalignant haematological diseases.

register data (as described below) were available. A case was considered confirmed if it met one of the diagnostic criteria listed in Table 1 in the presence of pneumonia as diagnosed by the treating physician.

Data were obtained from several sources. Since 1968, each resident in Denmark is assigned a unique personal identification number, and this number was used to crosslink between the different data sources.

Cases were identified from the national notification system. This database contains information on age, sex, how the diagnosis was made, travel history, and other exposures if known. Cases were classified as travel associated if the patient had spent at least one night away from home in the 10 days preceding disease onset and as hospital acquired if disease onset was less than 10 days from discharge or more than two days after admission. Data on treatment, underlying diseases, and other patient factors are not systematically collected through the notification system. For patients having several episodes of Legionnaires' disease, only the first was included.

Culture results for the period 1996 to 2006 were obtained from the database of the legionella reference laboratory at Statens Serum Institut.

Date of death was obtained from the civil registry, and death was considered possibly related to Legionnaires' disease if it occurred within 30 days of disease onset. If the date of onset was not stated on the notification form, date of hospitalization was used.

The national registry of hospital discharges holds information on all diagnoses listed on patients' forms at discharge from a hospital admission (classified according to the International classification of diseases (ICD) revision 10 (in use from 1994) or 8 (before 1994)). Both the principal diagnosis leading to hospitalization and any relevant comorbidity diagnoses should be registered at discharge, and so this register gives information not only on the diseases leading to hospitalization but also on underlying conditions. For each patient, diagnoses from the 5 years preceding and 1 month following onset of Legionnaires' disease were retrieved. The ICD codes were grouped according to 11 disease categories (listed in Table 2), and each patient was then assigned a comorbidity score based on the number of disease categories in which at least one diagnosis was recorded for that patient. Each disease category was counted only once for each patient, regardless of the number of diagnoses or discharges that a patient experienced within the same category. The comorbidity score could thus be between 0 (no discharge diagnoses registered in the five-year period) and 11 (at least one diagnosis in each category). Organ transplant recipients were grouped with the immune deficiency diseases as the accompanying immunosuppressive therapy was believed to 


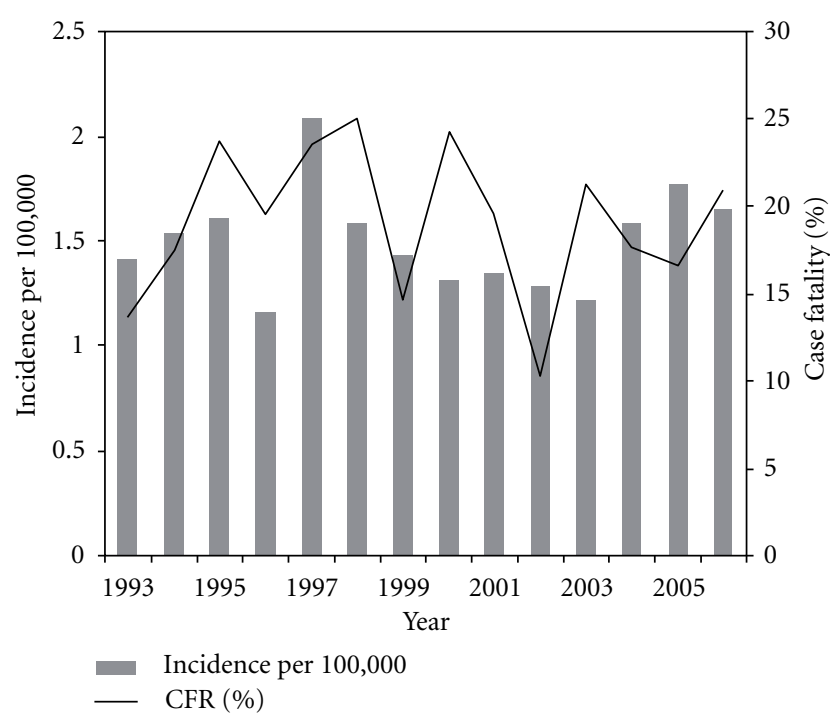

FIGURE 1: Incidence and case fatality rate (\%) of Legionnaires' disease in Denmark, 1993-2006 (confirmed cases only).

be the key risk factor for these patients. Similarly, HIV/AIDS was categorized with the immune deficiencies and not with infectious diseases.

A descriptive analysis of incidence, CFR, and comorbidities for all cases and for the different exposure categories was made as well as a description of the culture results. Furthermore, univariable and multivariable analyses of factors thought to be linked to the risk of dying from Legionnaires' disease were performed using logistic regression. The analyses were carried out on both the entire dataset and on a subgroup of patients for whom culture results were available. STATA was used in the analysis. The Danish Data Protection Board was notified of the study.

\section{Results}

During the study period, 1357 cases of Legionnaires' disease were notified, corresponding to an average annual incidence of 1.8 per 100000 population. In 11 cases, the patient had no Danish civil registration number (e.g., tourists) or the number was unavailable, and these cases were excluded as cross linking to other data sources was impossible. A further 230 cases were not laboratory confirmed by the criteria in Table 1, leaving 1116 cases for analysis (1.5 per 100000 population per year), Figure 1.

The median age was 58 years for males (range 15-95 years) and 62 years for females (range 13-92 years) and 66\% of the patients were male. Travel-associated Legionnaires' disease occurred in 302 (27\%) patients, 94\% of which had travelled abroad and 6\% in Denmark. Nosocomial transmission was reported for $166(15 \%)$ of the patients. The remaining $648(58 \%)$ of cases were considered community acquired. No large community outbreaks were registered during the study period.

At least one comorbidity diagnosis was recorded in 820 $(73 \%)$ of all cases, $188(62 \%)$ of travel-associated cases,
$471(73 \%)$ of community acquired cases, and $161(97 \%)$ of nosocomial cases (the remaining five $(3 \%)$ of the patients with hospital acquired disease were hospitalized and infected in orthopaedic or psychiatric wards and had none of the diagnoses included in the comorbidity score). The distribution of comorbidity diagnoses is shown in Table 2 . The proportion of patients having at least one comorbidity diagnosis increased with increasing age, Table 3.

Positive culture results from 1996 to 2006 were retrieved from the database of the national reference laboratory, in which culture results for 448 patients were available. Of these, 28 had not been notified, and one patient notified with two episodes was excluded because the comorbidity data referred to the first episode and the culture result to the second. The analysis was thus performed on 419 cases. Most of the isolates were L. pneumophila, with the dominant serogroups being $1(60 \%)$ and $3(23 \%)$, but with variations between exposure categories: travel-associated cases were almost exclusively caused by strains belonging to serogroup 1 , whereas several other serogroups were isolated from nosocomial cases.

The overall CFR was $19 \%$, ranging from $7 \%$ in travel associated cases to $37 \%$ in hospital-acquired cases. Culturepositive cases had a CFR of $30 \%$, lower for most subgroups of L. pneumophila serogroup 1, and higher for other serogroups and species (Table 4). No sex differences in mortality were observed for any of the age groups, exposure categories or risk factors. Table 5 shows CFR and results of univariable and multivariable analysis. Factors significantly related to an increased risk of death were age above 60 years, comorbidity score of at least 2 , and hospital acquisition of disease, whereas travel-associated cases had a lower case fatality.

Table 6 shows the results for only the culture positive cases including the results of culture in the analysis. Patients infected with $L$. pneumophila serogroup 6 had increased risk of death compared to those with other serogroups or species whereas travel-associated cases had lower case fatality.

\section{Discussion}

This study provides information on the incidence and mortality of Legionnaires' disease in Denmark, on factors linked to the risk of dying from the disease, including the influence of culture results.

4.1. Risk Factors for Death from Legionnaires' Disease. Patients with comorbidities in two or more disease categories, patients over 60 years, patients with hospital acquired disease, and patients infected by L. pneumophila serogroup 6 were at increased risk of dying, whereas patients with travelassociated disease had a lower risk.

Several other studies have investigated the factors related to the outcome of Legionnaires' disease. Some included small numbers of patients or only patients with severe disease and/or admitted to intensive care units [6-8] making comparison with our results difficult.

Others have been carried out in patient populations comparable to ours and have found similar results. Poupard et al. [9] examined the prognostic factors in patients aged 
TABLE 3: Distribution of risk factors by age groups. Confirmed notified cases of LD, Denmark 1993-2006.

\begin{tabular}{|c|c|c|c|c|c|}
\hline Age groups & At least one comorbidity no. (\%) & Travel associated no. (\%) & Nosocomial no. (\%) & Male sex no. (\%) & Total \\
\hline$<40$ years & $49(60 \%)$ & $19(23 \%)$ & $11(13 \%)$ & $58(71 \%)$ & 82 \\
\hline $40-59$ years & $324(64 \%)$ & $154(32 \%)$ & $47(10 \%)$ & $345(71 \%)$ & 487 \\
\hline $60-69$ years & $221(80 \%)$ & $88(32 \%)$ & $43(16 \%)$ & $165(60 \%)$ & 275 \\
\hline $70-79$ years & $164(82 \%)$ & $37(19 \%)$ & $50(25 \%)$ & $124(62 \%)$ & 199 \\
\hline $80+$ years & $62(85 \%)$ & $4(5.5 \%)$ & $15(21 \%)$ & $43(59 \%)$ & 73 \\
\hline All patients & $820(73 \%)$ & $302(27 \%)$ & $166(15 \%)$ & $735(66 \%)$ & 1116 \\
\hline
\end{tabular}

TABle 4: Distribution of species, serogroups, and subgroups for different exposure categories and case fatality ratio by infecting species/serogroup. Culture positive, notified cases, $N=419$.

\begin{tabular}{|c|c|c|c|c|}
\hline Species & All cases & Travel associated & Nosocomial & Deaths \\
\hline Legionella pneumophila & $403(96 \%)$ & $97(98 \%)$ & $71(93 \%)$ & $118(29 \%)$ \\
\hline Serogroup $1^{1}$ & $253(60 \%)$ & $85(86 \%)$ & $28(37 \%)$ & $60(24 \%)$ \\
\hline Olda/Oxford (n-P) & $61(15 \%)$ & $6(6.1 \%)$ & $21(28 \%)$ & $16(26 \%)$ \\
\hline Benidorm $(\mathrm{P})$ & $63(15 \%)$ & $30(30 \%)$ & 0 & $11(17 \%)$ \\
\hline Philadelphia (P) & $56(13 \%)$ & $26(26 \%)$ & $3(3.9 \%)$ & $10(18 \%)$ \\
\hline Knoxville (P) & $39(9 \%)$ & $15(15 \%)$ & 0 & $12(31 \%)$ \\
\hline Bellingham (n-P) & $20(5 \%)$ & $1(1.0 \%)$ & $4(5.3 \%)$ & $8(40 \%)$ \\
\hline Allentown/France (P) & $12(3 \%)$ & $6(6.1 \%)$ & 0 & $2(17 \%)$ \\
\hline Subgroup unknown & $2(0.5 \%)$ & $1(1 \%)$ & 0 & $1(50 \%)$ \\
\hline Serogroup 2 & $2(0.5 \%)$ & $1(1 \%)$ & 0 & $1(50 \%)$ \\
\hline Serogroup $3^{2}$ & $95(23 \%)$ & $7(7.1 \%)$ & $21(28 \%)$ & $34(36 \%)$ \\
\hline Serogroup 4 & $7(1.7 \%)$ & 0 & $2(2.6 \%)$ & $4(57 \%)$ \\
\hline Serogroup 5 & $7(1.7 \%)$ & 0 & $5(6.6 \%)$ & $3(43 \%)$ \\
\hline Serogroup 6 & $20(5 \%)$ & $4(4 \%)$ & $7(9.2 \%)$ & $11(55 \%)$ \\
\hline Serogroup 8 & $1(0.2 \%)$ & 0 & 0 & \\
\hline Serogroup 10 & $8(1.9 \%)$ & 0 & $4(5.3 \%)$ & $3(38 \%)$ \\
\hline Serogroup 12 & $3(0.7 \%)$ & 0 & $2(2.6 \%)$ & $1(33 \%)$ \\
\hline Serogroup 14 & $1(0.2 \%)$ & 0 & 0 & 0 \\
\hline Serogroup 15 & $5(1.1 \%)$ & 0 & $2(2.6 \%)$ & $1(20 \%)$ \\
\hline Serogroup unknown & $1(0.2 \%)$ & 0 & 0 & 0 \\
\hline Legionella bozemanii & $4(1.0 \%)$ & $2(2 \%)$ & 0 & $3(75 \%)$ \\
\hline Legionella dumoffii & $2(0.5 \%)$ & 0 & 0 & 0 \\
\hline Legionella longbeachae & $3(0.7 \%)$ & 0 & 0 & 0 \\
\hline Legionella micdadei & $7(1.7 \%)$ & 0 & $5(6.6 \%)$ & $3(43 \%)$ \\
\hline Total & $419(100 \%)$ & $99(100 \%)$ & $76(100 \%)$ & $124(30 \%)$ \\
\hline
\end{tabular}

${ }^{1}$ n-P: Non-Pontiac subgroups not reactive with MAb 3/1 of the Dresden panel and P: Pontiac subgroups reactive with MAb 3/1 of the Dresden panel.

${ }^{2} \mathrm{Few}(<5)$ cases in this group could belong to nonidentified serogroups.

under or over 60 years and found higher mortality in the latter group. Cancer, kidney disease, and alcohol abuse predisposed to death in both age groups. In the older age group, immune suppression, male gender, hospital acquisition, and being a nursing home-resident also increased the risk of dying, whereas travel-associated cases where less likely to die. Studies of notified, confirmed cases from Catalonia [10] and the United States [11] both found increasing age, hospitalacquired disease, immunosuppressive treatment, and cancer to predispose to death. In the American study, infection with L. pneumophila serogroup 6, male gender, and kidney disease also increased the risk of dying.
Another Danish study, covering a part of the country, analysed prognostic factors separately for community and hospital acquired disease [12]. For the former, delay in treatment with antibiotics active against legionellae, certain biochemical laboratory results, and markers for disease severity (factors comprised in PSI and/or CURB65 scores) were significantly related to risk of dying. When symptoms and laboratory results thought to be indicators of disease severity rather than true prognostic factors were excluded from the analysis, age above 65 years and Charlson comorbidity index score above two remained significant in the multivariable analysis. Two studies from the Netherlands [13] and Australia 
TABLE 5: Uni- and multivariable analyses of prognostic factors for confirmed, notified cases of Legionnaires' disease, Denmark $1993-2006$. $N=1116$.

\begin{tabular}{|c|c|c|c|c|}
\hline Prognostic factor & No. of cases & No. (\%) deaths & Univariable OR for death $(95 \% \mathrm{CI})$ & Multivariable OR for death $(95 \% \mathrm{CI})$ \\
\hline \multicolumn{5}{|l|}{ Age group } \\
\hline$<40$ years & 82 & $6(7 \%)$ & 1 (reference) & 1 (reference) \\
\hline $40-59$ years & 487 & $59(12 \%)$ & $1.7(0.7-4.2)$ & $1.8(0.7-4.5)$ \\
\hline $60-69$ years & 275 & $56(20 \%)$ & $3.2(1.3-7.8)$ & $3.0(1.2-7.5)$ \\
\hline $70-79$ years & 199 & $62(31 \%)$ & $5.7(2.4-14)$ & $4.5(1.8-11)$ \\
\hline $80+$ years & 73 & $33(45 \%)$ & $10(4.0-27)$ & $8.3(3.1-22)$ \\
\hline \multicolumn{5}{|l|}{ Gender } \\
\hline Male & 735 & $141(19 \%)$ & 1 (reference) & 1 (reference) \\
\hline Female & 381 & $75(20 \%)$ & $1.0(0.8-1.4)$ & $0.8(0.6-1.1)$ \\
\hline \multicolumn{5}{|l|}{ Comorbidity score } \\
\hline 0 & 296 & $25(8 \%)$ & 1 (reference) & 1 (reference) \\
\hline 1 & 309 & $46(15 \%)$ & $1.9(1.1-3.2)$ & $1.5(0.9-2.6)$ \\
\hline 2 & 220 & $48(22 \%)$ & $3.0(1.8-5.1)$ & $2.0(1.1-3.4)$ \\
\hline 3 & 143 & $44(31 \%)$ & $4.8(2.8-8.3)$ & $3.4(1.9-6.0)$ \\
\hline 4 & 77 & $26(34 \%)$ & $5.5(3.0-10)$ & $3.3(1.7-6.4)$ \\
\hline 5 or more & 71 & $27(38 \%)$ & $6.7(3.5-12)$ & $3.5(1.8-6.9)$ \\
\hline \multicolumn{5}{|l|}{ Nosocomial } \\
\hline No & 950 & $155(16 \%)$ & 1 (reference) & 1 (reference) \\
\hline Yes & 166 & $61(37 \%)$ & $3.0(2.0-4.3)$ & $1.6(1.0-2.3)$ \\
\hline \multicolumn{5}{|l|}{ Travel-associated } \\
\hline No & 814 & $194(24 \%)$ & 1 (reference) & 1 (reference) \\
\hline Yes & 302 & $22(7 \%)$ & $0.3(0.2-0.4)$ & $0.4(0.2-0.6)$ \\
\hline
\end{tabular}

[14] investigated prognostic factors in patients related to outbreaks. In the Dutch study, smoking, alcohol abuse, and factors related to the severity of pneumonia (such as temperature above $38.5^{\circ} \mathrm{C}$ and bilateral lung involvement) increased the risk of death or admission to intensive care unit, as did delay in antibiotic treatment. Among the Australian patients, multivariable analysis was not performed because only few deaths occurred, but in univariate analysis heart failure and kidney disease increased risk of dying. Smokers had a longer duration of illness but not a higher mortality than nonsmokers.

In summary, the prognostic factors identified in this study are well in accordance with results from other studies. For the hospital acquired cases, our study found a high OR in univariable analysis, but when controlling for comorbidity score and age this was much less marked, suggesting that these factors explain most of the excess mortality.

The lower mortality among travel-associated cases has been attributed to better health condition of persons that are able to travel or to differences in infecting legionellae strains [9]. In a study examining risk factors for acquiring Legionnaires' disease, travellers were found to have less comorbidity than other patients [15]. In our study, as well as that by Poupard et al. [9], the lower mortality remained, despite controlling for comorbidities, but the severity of the conditions could not be taken into account and may still be important, as people with less severe forms of any underlying condition may be more likely to travel. It may also be of importance that travelling is a well-known risk factor for Legionnaires' disease, leading to testing of more patients, including those with mild disease for which prognosis is probably better.

4.2. Culture Results. The distribution of infecting Legionella species and serogroups, with the majority being L. pneumophila, is similar to other reports, although the serogroup distribution shows less cases of serogroup 1 and more serogroup 3 compared to other reports $[1,16,17]$.

Culture positive cases had a high CFR. This may be due to the fact that more severe cases are more likely to have a positive culture [1] or to the fact that diagnosis by culture takes longer time than by UAT. Benin et al. [16] found cases that were culture positive and had either negative UAT or no UAT performed to be significantly more likely to die. This could reflect a lower virulence of serogroup 1 or the fact that these cases can be diagnosed-and thus treated-early by UAT. In Denmark, PCR is commonly used and allows early diagnosis of UAT-negative cases; diagnosis only by culture is thus rare and cannot explain the higher mortality for other species and serogroups.

Patients infected with L. pneumophila serogroup 6 were more likely to die than those with other serogroups or species. Marston et al. [11] also found a high risk of death with serogroup 6 in a multivariable analysis adjusting for nosocomial acquisition, underlying diseases and age and 
TABLE 6: Uni- and multivariable analyses of prognostic factors for culture-confirmed, notified cases of Legionnaires' disease, Denmark $1996-2006, N=419$

\begin{tabular}{|c|c|c|c|c|}
\hline Prognostic factor & No. of cases & No. (\%) deaths & $\begin{array}{l}\text { Univariable OR for death } \\
(95 \% \mathrm{CI})\end{array}$ & $\begin{array}{c}\text { Multivariable OR for death } \\
(95 \% \mathrm{CI})\end{array}$ \\
\hline \multicolumn{5}{|l|}{ Age group } \\
\hline$<40$ years & 18 & $4(22 \%)$ & 1 (reference) & 1 (reference) \\
\hline $40-59$ years & 176 & $35(20 \%)$ & $0.9(0.3-2.8)$ & $0.9(0.3-3.0)$ \\
\hline $60-69$ years & 101 & $32(32 \%)$ & $1.6(0.5-5.3)$ & $1.8(0.5-6.3)$ \\
\hline $70-79$ years & 92 & $33(36 \%)$ & $2.0(0.6-6.4)$ & $1.9(0.6-6.8)$ \\
\hline $80+$ years & 32 & $20(63 \%)$ & $5.8(1.6-22)$ & $5.5(1.3-22)$ \\
\hline \multicolumn{5}{|l|}{ Gender } \\
\hline Male & 283 & $83(29 \%)$ & 1 (reference) & 1 (reference) \\
\hline Female & 136 & $41(30 \%)$ & $1.0(0.7-1.6)$ & $0.8(0.5-1.2)$ \\
\hline \multicolumn{5}{|l|}{ Comorbidity score } \\
\hline 0 & 66 & $14(21 \%)$ & 1 & 1 \\
\hline 1 & 105 & $26(25 \%)$ & $1.2(0.6-2.6)$ & $1.0(0.5-2.3)$ \\
\hline 2 & 94 & $30(32 \%)$ & $1.7(0.8-3.6)$ & $1.3(0.6-3.0)$ \\
\hline 3 & 59 & $19(32 \%)$ & $1.8(0.8-4.0)$ & $1.8(0.7-4.2)$ \\
\hline 4 & 48 & $18(38 \%)$ & $2.2(0.9-5.1)$ & $1.6(0.6-4.0)$ \\
\hline 5 or more & 47 & $17(36 \%)$ & $2.1(0.9-4.9)$ & $1.4(0.5-3.4)$ \\
\hline \multicolumn{5}{|l|}{ Nosocomial } \\
\hline No & 343 & $96(28 \%)$ & 1 (reference) & 1 (reference) \\
\hline Yes & 76 & $28(37 \%)$ & $1.5(0.9-2.5)$ & $0.8(0.5-1.6)$ \\
\hline \multicolumn{5}{|l|}{ Travel-associated } \\
\hline No & 320 & $112(35 \%)$ & 1 (reference) & 1 (reference) \\
\hline Yes & 99 & $12(12 \%)$ & $0.3(0.1-0.5)$ & $0.3(0.1-0.6)$ \\
\hline \multicolumn{5}{|l|}{ Culture result } \\
\hline $\begin{array}{l}\text { L pneumophila serogroup } 1 \text {, Pontiac } \\
\text { (MAb 3/1 pos) }\end{array}$ & 172 & $36(21 \%)$ & 1 (reference) & 1 (reference) \\
\hline $\begin{array}{l}\text { L pneumophila serogroup } 1 \\
\text { non-Pontiac (Mab 3/1 neg) }\end{array}$ & 81 & $24(30 \%)$ & $1.6(0.9-2.9)$ & $0.8(0.4-1.7)$ \\
\hline L pneumophila serogroup 3 & 95 & $34(36 \%)$ & $2.1(1.2-3.7)$ & $1.1(0.6-2.1)$ \\
\hline L pneumophila serogroup 6 & 20 & $11(55 \%)$ & $4.6(1.8-12)$ & $3.1(1.1-9.0)$ \\
\hline L pneumophila, other & 35 & $13(37 \%)$ & $2.2(1.0-4.9)$ & $1.0(0.4-2.4)$ \\
\hline Nonpneumophila & 16 & $6(38 \%)$ & $2.3(0.8-6.7)$ & $1.4(0.5-4.5)$ \\
\hline
\end{tabular}

sex of the patients. The reason for this may be differences in virulence between different species and serogroups of legionellae [11]. Differences in mortality were also noted between different subgroups of $L$. pneumophila serogroup 1. A study comparing patients infected with different strains of L. pneumophila serogroup 1 found differences both in terms of characteristics of the patients infected with different strains and their mortality [18]. In our study, the excess mortality for L. pneumophila serogroup 1 non-Pontiac, serogroup 3, and other serogroups could be explained by controlling for patient characteristics (Table 6), but the high OR for Serogroup 6 remained in multivariable analysis, suggesting that this serogroup may indeed be more virulent.

It is worth noting that the proportion of culture positive cases $(38 \%)$ is very high compared to the European average of $8.9 \%$ in $2005 / 06$ [19].
4.3. Incidence and Mortality. The average incidence of Legionnaires' disease in Europe has increased from 4.1 per million population in 1993 to 11.2 per million in 2006 [19], with the incidence in 2006 varying between less than 1 per million and 28.4 per million between countries. At 23.4 cases per million in 2006 (including cases not considered confirmed for the purpose of the present study), the incidence in Denmark is relatively high [19].

The mortality found in this study is relatively high compared to the mortality reported from other countries $[2,9,10,13,16,20,21]$. Furthermore, a decline in mortality has been observed in several countries in the past 3 decades [2], with a decrease from $34 \%$ in 1985 to $11.5 \%$ in 1998 in the United States [16] and from 35\% in 1993 to $5.6 \%$ in 2004 in Spain [10]; a similar decline cannot be observed in these data (Figure 1). In some studies, only death attributed specifically 
to Legionnaires' disease was included [16, 21], which would make the observed fatality rate lower than in ours.

The decrease in mortality observed in other countries has been attributed to a number of reasons: better diagnostics, particularly the use of UAT $[2,10,16,21]$, changes in treatment guidelines leading to more patients receiving antibiotics with activity against legionellae as empiric treatment for pneumonia $[2,16,21]$, better detection of outbreaks resulting in more cases being identified and adequate treatment being given early in the course of illness $[10,22]$, and heightened awareness of the disease among clinicians [10]. CFRs have been lower in outbreak settings than among sporadic community acquired cases $[10,22]$, and in a comparison of mortality during outbreaks in different countries, lower mortality was observed in countries using legionella-active antibiotics more commonly as empiric treatment for pneumonia, although differences in time to detection of the outbreaks may also have played a role [16, 23].

The reasons for the higher mortality in Denmark during the study period cannot be determined from the present data, but several factors may play a role: the overall mortality in countries where large community outbreaks occur may be lower due to the lower mortality of the outbreak related cases. Most cases of community acquired pneumonia in Denmark receive penicillin as the initial treatment and legionellaactive antibiotics are only prescribed when the diagnosis is made or if there is no response to penicillin. Diagnosis for Legionnaires' disease is not routinely performed but based on clinical suspicion which may result in only the more severe cases with higher mortality being recognized. In a study from Catalonia where all hospitalized patients with community acquired pneumonia were systematically tested for legionella infection, regardless of the severity of the pneumonia, the CFR was 5\% [20].

Furthermore, among the patients in the present study a higher proportion had at least one comorbidity than in other studies $[9,13,20]$, suggesting that the diagnosis of Legionnaires' disease is sought mainly in patients with predisposing conditions for whom the prognosis is worse. Correspondingly, the increasing incidence seen in other countries, as a result of increased diagnostic activity, improved diagnostics and identification of milder cases, has not been seen in Denmark.

4.4. Strengths and Limitations. The strengths of the study are the large number of patients studied, the availability of data from several different registers, and the availability of culture results for a high proportion of cases.

However, the study also has several limitations. The use of discharge diagnoses to define comorbidities means that milder forms of disease, not in themselves leading to hospitalization, will only be included if the patient is hospitalized for another cause and if all comorbidity diagnoses are correctly listed at discharge. As most of the patients were hospitalized for the Legionnaires' disease episode, the impact of this may be lessened.

Other factors known to be risk factors for acquisition of Legionnaires' disease, and possibly also for dying from the disease, such as smoking or high daily alcohol intake, could not be studied or only indirectly through diagnoses for other diseases caused by the same risk factor, such as chronic obstructive lung disease. However, another study found only smoking and not smoking related disorders to be a risk factor for the acquisition of the disease [15] so, possibly, these conditions cannot be used as a substitute for the risk factor itself. An ICD diagnosis for alcohol abuse does exist but applies to severe cases, and most patients with moderate or even high daily consumption would not be included. Furthermore, we have no data on treatment, neither of the underlying disorders nor of Legionnaires' disease, so the effect of, for instance, immunosuppressive treatment, or of delays in appropriate antibiotic treatment cannot be studied.

The study includes only notified cases so there may be underreporting. Misclassification of cases based on errors on the reporting form probably also occurs. For example, 18 of the cases reported as culture positive to the Department of Epidemiology had no culture result in the reference laboratory database. This may be due to the local microbiology laboratory not sending isolates to the reference laboratory but may also reflect reporting errors or misreporting.

\section{Conclusion}

This national study has provided further evidence for the importance of several prognostic factors in Legionnaires' disease and gives further insight into the variation in disease outcome according to the infecting species and serogroups. A prospective study of pneumonia patients with more systematic testing for legionellae and recording of all personal risk factors, less severe underlying diseases, and immunosuppressive treatment etcetera would be valuable to gain more knowledge on these issues. This could also help define patients at high risk of dying, for whom a different treatment strategy - such as including a legionellae-active antibiotic in the empiric treatment of pneumonia and then discontinuing this if another cause of pneumonia is confirmed-could be adopted.

The mortality of Legionnaires' disease in Denmark is high compared to other countries, underlining the need for timely and appropriate diagnosis and treatment, and for a heightened degree of awareness of the disease, including in cases not thought to be "typical" or "suspicious."

The finding of a high mortality for L. pneumophila serogroup 6, which cannot be diagnosed by UAT, and Benin et al. [16] finding of a higher mortality among patients that had positive cultures but negative UAT point to the relevance of diagnostics-for example, PCR - that can detect other serogroups than the UATs currently in use, and that are faster and more sensitive than culture.

\section{References}

[1] B. M. W. Diederen, “Legionella spp. and Legionnaires' disease," Journal of Infection, vol. 56, no. 1, pp. 1-12, 2008.

[2] J. Carratala and C. Garcia-Vidal, "An update on Legionella," Current Opinion in Infectious Diseases, vol. 23, no. 2, pp. 152-157, 2010. 
[3] H. von Baum, S. Ewig, R. Marre et al., "Community-acquired Legionella pneumonia: new insights from the German competence network for community acquired pneumonia," Clinical Infectious Diseases, vol. 46, no. 9, pp. 1356-1364, 2008.

[4] R. B. Brown, "Legionella pneumonia: the spectrum continues to expand," Chest, vol. 125, no. 6, pp. 1979-1980, 2004.

[5] C. W. Olsen, P. Elverdal, C. S. Jørgensen, and S. A. Uldum, "Comparison of the sensitivity of the Legionella urinary antigen EIA kits from Binax and Biotest with urine from patients with infections caused by less common serogroups and subgroups of Legionella," European Journal of Clinical Microbiology and Infectious Diseases, vol. 28, no. 7, pp. 817-820, 2009.

[6] M. El-Ebiary, X. Sarmiento, A. Torres et al., "Prognostic factors of severe Legionella pneumonia requiring admission to ICU," American Journal of Respiratory and Critical Care Medicine, vol. 156, no. 5, pp. 1467-1472, 1997.

[7] A. Gacouin, Y. Le Tulzo, S. Lavoue et al., "Severe pneumonia due to Legionella pneumophila: prognostic factors, impact of delayed appropriate antimicrobial therapy," Intensive Care Medicine, vol. 28, no. 6, pp. 686-691, 2002.

[8] L. S. Tkatch, S. Kusne, W. D. Irish, S. Krystofiak, and E. Wing, "Epidemiology of Legionella pneumonia and factors associated with Legionella-related mortality at a tertiary care center," Clinical Infectious Diseases, vol. 27, no. 6, pp. 1479-1486, 1998.

[9] M. Poupard, C. Campèse, P. Bernillon, and D. Che, "Factors associated with mortality in Legionnaires' disease, France, 2002-2004," Medecine et Maladies Infectieuses, vol. 37, no. 6, pp. 325-330, 2007.

[10] A. Dominguez, J. Alvarez, M. Sabria et al., "Factors influencing the case-fatality rate of Legionnaires' disease," International Journal of Tuberculosis and Lung Disease, vol. 13, no. 3, pp. 407-412, 2009.

[11] B. J. Marston, H. B. Lipman, and R. F. Breiman, "Surveillance for legionnaires' disease: risk factors for morbidity and mortality," Archives of Internal Medicine, vol. 154, no. 21, pp. 2417-2422, 1994.

[12] S. Jespersen, O. S. Søgaard, H. C. Schønheyder, M. J. Fine, and L. Østergaard, "Clinical features and predictors of mortality in admitted patients with community- and hospital-acquired legionellosis: a Danish historical cohort study," BMC Infectious Diseases, vol. 10, article 124, 2010.

[13] K. D. Lettinga, A. Verbon, G. J. Weverling et al., "Legionnaires' disease at a Dutch flower show: prognostic factors and impact of therapy," Emerging Infectious Diseases, vol. 8, no. 12, pp. 1448-1454, 2002.

[14] B. P. Howden, R. L. Stuart, G. Tallis, M. Bailey, and P. D. R. Johnson, "Treatment and outcome of 104 hospitalized patients with legionnaires' disease," Internal Medicine Journal, vol. 33, no. 11, pp. 484-488, 2003.

[15] J. W. D. Boer, J. Nijhof, and I. Friesema, "Risk factors for sporadic community-acquired Legionnaires' disease. A 3-year national case-control study," Public Health, vol. 120, no. 6, pp. 566-571, 2006.

[16] A. L. Benin, R. F. Benson, and R. E. Besser, "Trends in legionnaires disease, 1980-1998: declining mortality and new patterns of diagnosis," Clinical Infectious Diseases, vol. 35, no. 9 , pp. 1039-1046, 2002.

[17] J. H. Helbig, S. Bernander, M. Castellani Pastoris et al., "PanEuropean study on culture-proven Legionnaires' disease: distribution of Legionella pneumophila serogroups and monoclonal subgroups," European Journal of Clinical Microbiology and Infectious Diseases, vol. 21, no. 10, pp. 710-716, 2002.
[18] C. Ginevra, A. Duclos, P. Vanhems et al., "Host-related risk factors and clinical features of community-acquired legionnaires disease due to the Paris and Lorraine endemic strains, 1998-2007, France," Clinical Infectious Diseases, vol. 49, no. 2, pp. 184-191, 2009.

[19] K. D. Ricketts and C. A. Joseph, "Legionnaires disease in Europe: 2005-2006," Euro Surveillance, vol. 12, no. 12, article 4, 2007.

[20] A. Mykietiuk, J. Carratalà, N. Fernández-Sabé et al., "Clinical outcomes for hospitalized patients with Legionella pneumonia in the antigenuria era: the influence of levofloxacin therapy," Clinical Infectious Diseases, vol. 40, no. 6, pp. 794-799, 2005.

[21] N. Sopena, L. Force, M. L. Pedro-Botet et al., "Sporadic and epidemic community legionellosis: two faces of the same illness," European Respiratory Journal, vol. 29, no. 1, pp. 138-142, 2007.

[22] J. Alvarez, A. Domínguez, M. Sabrià et al., "Impact of the Legionella urinary antigen test on epidemiological trends in community outbreaks of legionellosis in Catalonia, Spain, 1990-2004," International Journal of Infectious Diseases, vol. 13, no. 6, pp. e365-e370, 2009.

[23] O. Tello, C. Pelaz, A. Garcia-Fulgueiras, C. Joseph, J. Kool, J. Lee et al., "Update on the outbreak of legionnaires' disease in Murcia, Spain," Eurosurveillance, vol. 5, no. 29, article 1714, 2001. 


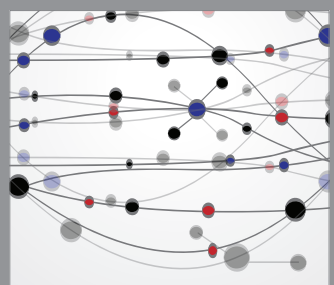

The Scientific World Journal
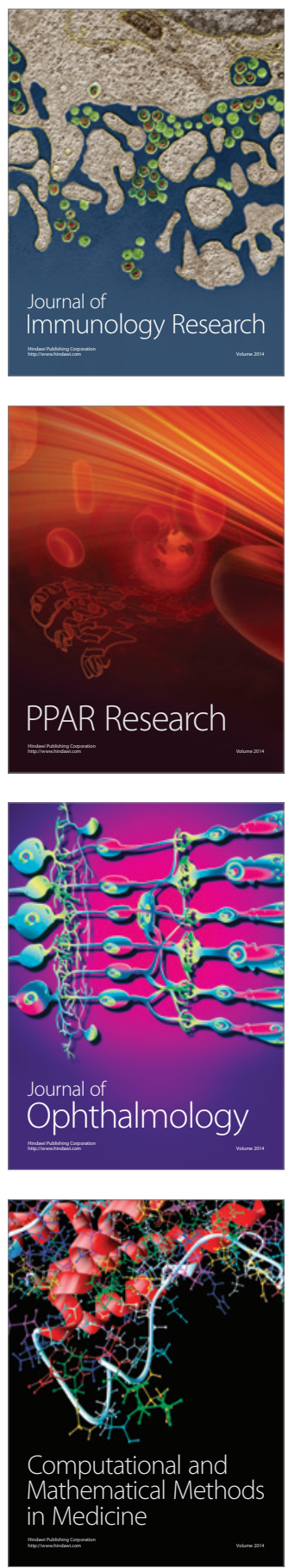

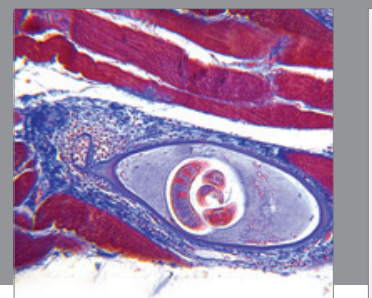

Gastroenterology

Research and Practice
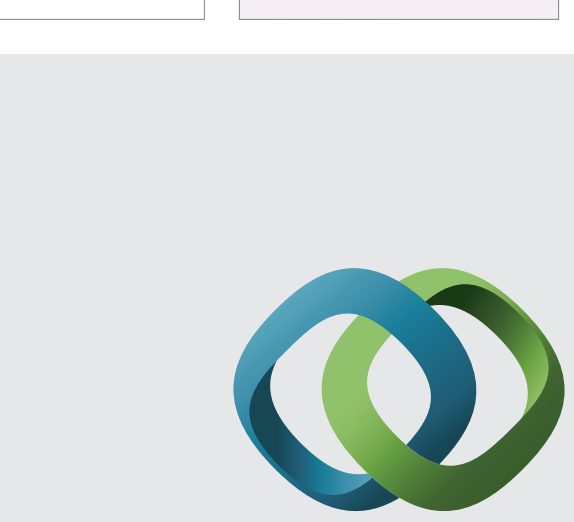

\section{Hindawi}

Submit your manuscripts at

http://www.hindawi.com
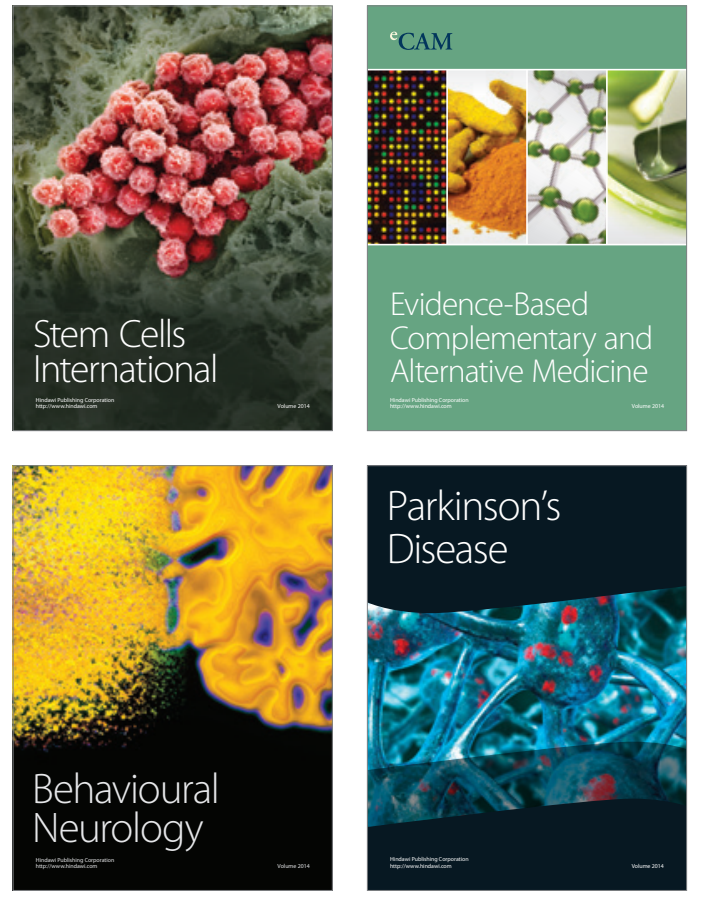
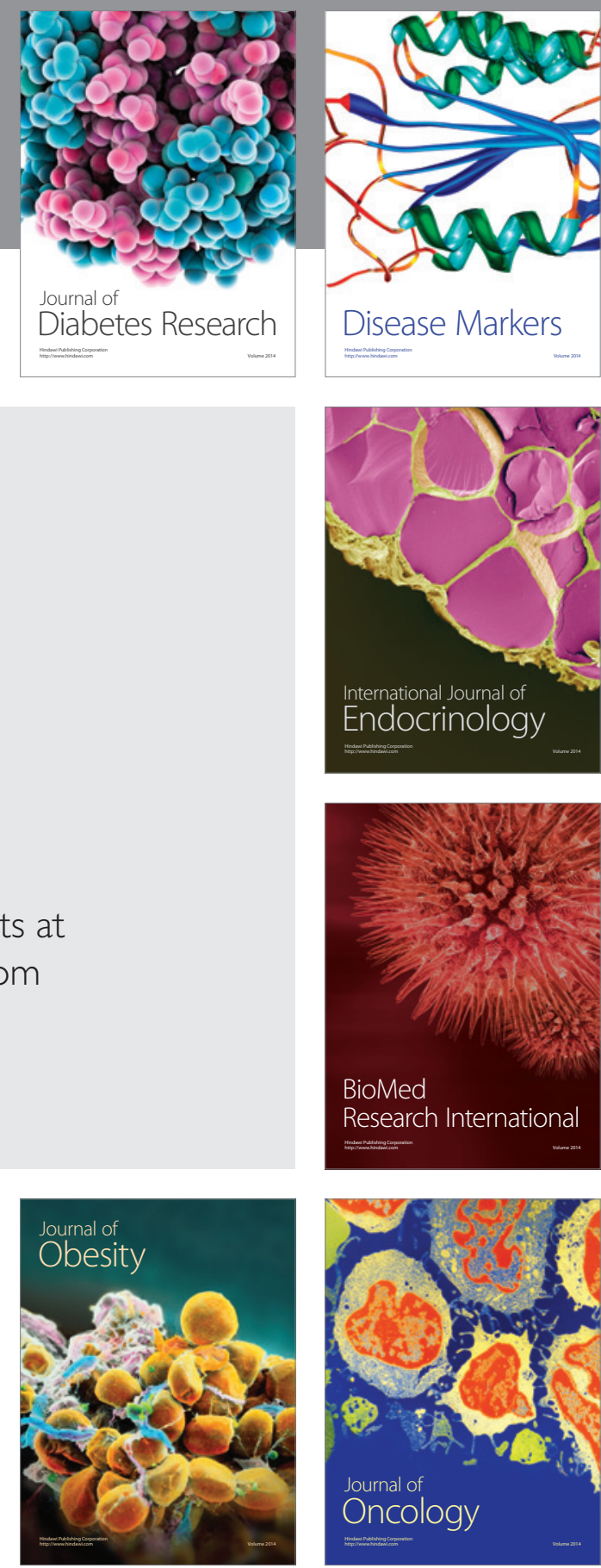

Disease Markers
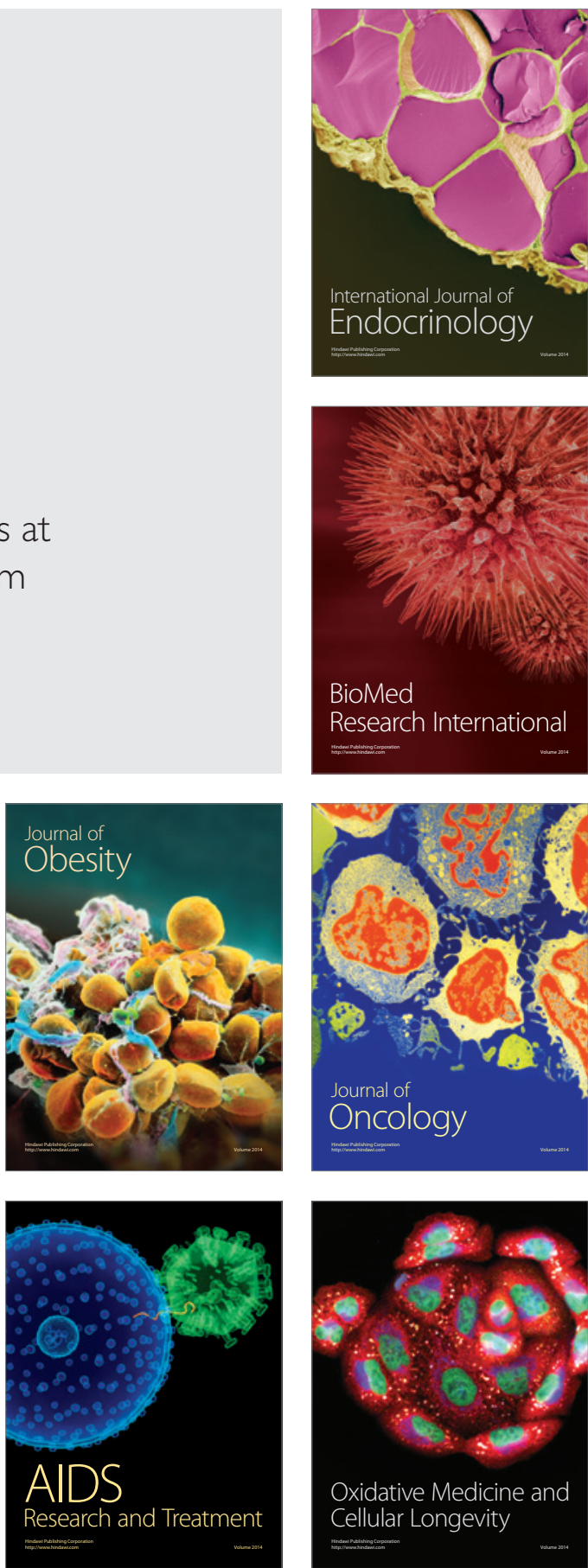